\title{
Bioinspired Nanoparticle Spray-Coating for Superhydrophobic Flexible Materials with Oil/Water Separation Capabilities
}

\author{
Nicasio R Geraldi ${ }^{1}$, Linzi E Dodd ${ }^{2}$, Ben B Xu ${ }^{1}$, David Wood ${ }^{2}$, Gary G Wells ${ }^{1}$, Glen \\ McHale $^{1}$, and Michael I Newton ${ }^{3}$ \\ ${ }^{1}$ Smart Materials and Surfaces Laboratory, Faculty of Engineering and Environment, \\ Northumbria University, Newcastle upon Tyne NE1 8ST, U.K. \\ ${ }^{2}$ Microsystems Technology Group, School of Engineering \& Computing Sciences, Durham \\ University, Durham DH1 3LE, U.K. \\ ${ }^{3}$ School of Science and Technology, Nottingham Trent University, Nottingham NG11 8NS, \\ $U . K$.
}

Corresponding Author: nicasio.geraldi@ northumbria.ac.uk

\begin{abstract}
Much of the inspiration for the creation of superhydrophobic surfaces has come from nature, from plant such as the Sacred Lotus (Nulembo nucifera), where the micro-scale papillae epidermal cells on the surfaces of the leaves are covered with nano-scale epicuticular wax crystalloids. The combination of the surface roughness and the hydrophobic wax coating produces a superhydrophobic wetting state on the leaves allowing them to self-clean and easily shed water. Here a simple scale-up carbon nanoparticle spray coating is presented that mimics the surface of the Sacred Lotus leaves and can be applied to a wide variety of materials, complex structures, and flexible substrates, rendering them superhydrophobic, with contact angles above $160^{\circ}$. The sprayable mixture is produced by combining toluene, polydimethylsiloxane (PDMS), and
\end{abstract}


inherently hydrophobic rapeseed soot. The ability to spray the superhydrophobic coating allows for the hydrophobisation of complex structures such a metallic meshes, which allows for the production of flexible porous superhydrophobic materials that when formed into U-shape channels, can be used to direct flows. The porous meshes, whilst being superhydrophobic, are also oleophilic. Being both superhydrophobic and oleophilic allows oil to pass through the mesh, whilst water remains on the surface. The meshes were tested for their ability to separate mixtures of oil and water in a flow situation. When silicone oil/water mixtures were passed over the meshes, all meshes tested were capable of separating more than $93 \%$ of the oil from the mixture.

\section{Introduction}

The ability to mimic naturally occurring superhydrophobic plant leaves, such as those of the Sacred Lotus (Nulembo nucifera) or those of Tuscan Kale (Brassica oleracea var. palmifolia DC), allows us to take advantage of their anti-wetting, self-cleaning, and anti-fouling capabilities [1,2]. The Sacred Lotus is able to produce superhydrophobic leaves through a combination of surface microstructures, papillae epidermal cells, and hydrophobic nano-roughness, epicuticular wax crystalloids [3]. The combination of the hierarchical roughness and hydrophobic wax is responsible for the superhydrophobicity. These properties mean that dirt and other contaminants that are deposited on the leaves are washed off by the motion of rain or dew droplets. In order to take advantage of these wetting properties it is important to understand how liquids behave of these surfaces. The basic laws of wetting for flat homogeneous surfaces were established by Young at the beginning of the 19th century[4]. It was not until the papers of Wenzel[5] (1936) and Cassie[6] (1944) were published that the governing properties of superhydrophobicity was developed. Wenzel proposed that when a liquid wets the contours of a surface, the roughness amplifies the 
tendency of the liquid to spread in the hydrophilic state or to enhance the non-wetting in the hydrophobic state. However, when it is energetically favorable for a liquid to bridge between the tops of surface features, the Cassie-Baxter state will be present, where the liquid sits atop of the surface features with air gaps present below the liquid. It is the Cassie-Baxter state that is responsible for superhydrophobicity, where droplets on surfaces exhibit apparent contact angles greater than $150^{\circ}$ and sliding angles less than $10^{\circ}$. The fabrication of superhydrophobic surfaces has been of great interest since the mid-1990's and has taken much inspiration from nature, especially from the Sacred Lotus, with the effect sometimes being referred to as the "Lotus effect" [7]. Onda et al. first demonstrated the ability to produce superhydrophobic fractal alkylketene dimers surfaces with contact angles as high as $174^{\circ}$ [8]. Since then, many different way have been devised to add surface structure suitable for superhydrophobic applications, including electrospinning of fibers[9], phase separation[10], crystal growth[11], etching[12], diffusion limited growth[12], electrodeposition[13], lithography[14], templating[15] and particle deposition[16]. These methods have a range of costs and complexity of process, and all are challenged when needing to achieve large surface area coverage. The ability to form superhydrophobic surfaces through particle deposition allows for larger area coverage and works well with simple spray coating methods. Current techniques include the spraying of modified metal and silica nanoparticles with functionalized coatings[17-21]. One issue with these processes is that the particles and powders can be costly, with the particles requiring further functionalizing to improve their intrinsic hydrophobicity and achieve a superhydrophobic coating.

Oil/water separation is an important process for the petrochemical, textiles and food industries. There is an increasing demand for effective inexpensive methods for the separation of waste products or oil spill recovery to reduce the environmental impact of contaminated water. It has 
been of interest to many researchers in the field, with inspiration coming from nature, from plants such as aquatic ferns (Salvinia) that have superhydrophobic/superoleophilic leaves and are capable of absorbing low surface tension oils, whilst repelling water [22]. In order to mimic this behavior in order to solve the oil/water separation problem, the use of porous materials with hierarchical surface roughness have been employed, which takes advantage of interfacial differences between the liquids and their wetting properties with the surface. The process relies on the preferential wetting of the surface by either of the liquids whilst the other is repelled. For this reason a porous material where the surface can be modified to include hierarchical structures offers the opportunity to selectively allow liquids with a certain range of surface tensions to pass[23-37]. The limitations of current technologies for the production of porous oil/water separating materials include small area production size, costly chemical processes, and complicated or costly production methods.

In this paper, a simple carbon nanoparticle (CNP) spray coating is presented, that can be applied to a wide variety of materials, conformable surfaces and to complex structures. The ability to coat flexible conformable surfaces offers the opportunity to form channels and tubes that can be used to direct flows. The use of carbon nanoparticles significantly reduces the cost of the coating. CNPs are readily available as a by-product of industrial processes and, chosen appropriately, do not require further functionalization, as the material is inherently hydrophobic[38-40].

\section{Methods}

The main constituent of the spray coating mixture, that is responsible for making the surface superhydrophobic, is carbon. The CNPs in this work were produced using a flame synthesis process, where rapeseed oil is made to combust in an oxygen-starved environment. This results in the production of intrinsically hydrophobic carbon nanoparticles in the form of soot, that need no 
further chemical functionalization[41]. The CNPs have an average size of $115 \pm 13 \mathrm{~nm}$, measured using scanning electron microscopy (SEM).

A mixture of toluene (Sigma Aldrich), rapeseed soot and polydimethylsiloxane (PDMS) (Sylgard 184, Dow Corning) formed the spray coating mixture. The constituents had a ratio of 230:3:2, respectively.

Firstly, the rapeseed soot was rinsed through a \#250 stainless steel mesh with the toluene. The mesh has a wire diameter of $40 \pm 2 \mu \mathrm{m}$ and a wire separation of $65 \pm 2 \mu \mathrm{m}$. This mixture was sonicated, using an Ultrawave U100 at room temperature, for 30 minutes to break up any clusters of soot remaining in the mixture and disperse the particles in the suspension. The PDMS, in a 10:1 wt/wt. elastomer to curing agent ratio was mixed, degassed for 30 minutes and added to the toluene/soot mixture. This final mixture was then stirred, using a magnetic stirrer, for a further 30 minutes.

The coating was applied using a Neo for Iwata CN gravity feed airbrush with a $5 \mathrm{ml}$ cup, attached to a DHCL solutions 3 bar compressor. The nozzle of the airbrush was positioned a distance of 10 $\mathrm{cm}$ from the surface and travelled in reciprocating vertical motion in order to evenly coat the surfaces. The surfaces were heated to $80{ }^{\circ} \mathrm{C}$ for $12 \mathrm{hrs}$ to ensure the PDMS was fully cured (Fig. 1). 


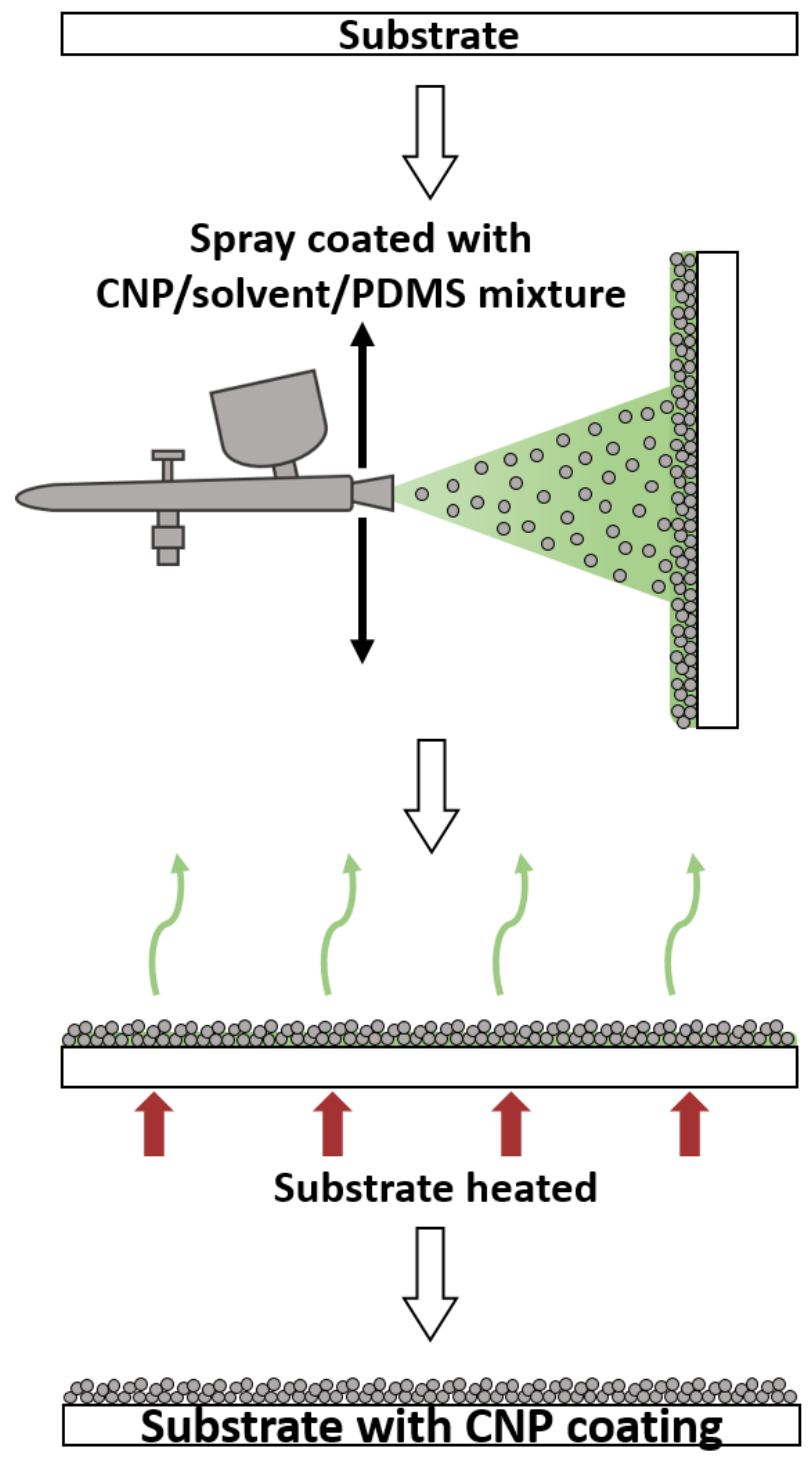

Fig 1. Schematic of the spray coating process.

To characterize the surfaces, drop shape analysis was performed using a Krüss DSA100. SEM was also performed, using a Tescan MIRA3, to image the top surface and the cross-section of the CNP coating (Fig 2).

For the oil/water separation experiments, six sizes of stainless steel mesh (The Mesh Company (Warrington) Ltd) were tested. These include \#50, \#98, \#150, \#250, \#400 and \#500, where the value indicates the number of wires per inch of the material (Fig 3(a)). The largest mesh, \#50, has 
a wire diameter of $193 \pm 4 \mu \mathrm{m}$ and a wire separation of $317 \pm 10 \mu \mathrm{m}$, whereas the smallest mesh, $\# 500$, has a wire diameter of $24 \pm 1 \mu \mathrm{m}$ and a wire separation of $29 \pm 2 \mu \mathrm{m}$. Mesh was chosen for its flexibility and ability to conform to specific shapes. To make the test samples, $300 \mathrm{~mm}$ by 100 mm sections of each mesh were suspended vertically. $10 \mathrm{ml}$ of the spray coating mixture was applied to each side of the mesh, $5 \mathrm{ml}$ at a time, with a 5 minute wait time between each $5 \mathrm{ml}$ coating. This wait time allowed the toluene to evaporate from the surface before the next coating was applied. After spraying, the mesh was then heated to $80^{\circ} \mathrm{C}$ for $12 \mathrm{hrs}$ (Fig 3(b)). Post-baking, a $100 \mathrm{~mm}$ by $50 \mathrm{~mm}$ section of each mesh was cutout, from which the u-shape channel with a fold line at the mid-point along its length was fashioned.

To test the superhydrophobic mesh surfaces for their ability to separate oil/water mixtures, $20 \mathrm{~g}$ silicone oil (20 cSt, Sigma Aldrich) and $20 \mathrm{~g}$ distilled water containing a small amount of watersoluble blue dye $(0.5 \mathrm{~g})$ was used. The separating ability of the mesh was tested in a flow situation as the mixture passed over the mesh. As the mixture travels over the mesh, the oil adheres and passes through the porous mesh, whilst the water continues to flow over the surface. The U-shape mesh channel was mounted horizontally above two empty containers of known mass, one to collect the separated oil and another to collect any run-off from the top surface of the mesh channel (Fig 3(c)). The oil/water mixture was dispensed onto the surface of the mesh from a height of $5 \mathrm{~mm}$ above the surface. After all the mixture passed over the surface, the mass of the oil container was recorded. To test the robustness of the separation process the surfaces where subjected to 5 cycles of the oil/water separation tests.

To test the stability of the prepared surfaces under corrosive environments, samples of the \#400 stainless steel coated mesh were immersed in $3.5 \%$ wt $\mathrm{NaCl}, 1 \mathrm{M} \mathrm{HCl}$, and $1 \mathrm{M} \mathrm{KOH}$ solutions, with their advancing and receding contact angles being measure over time. 

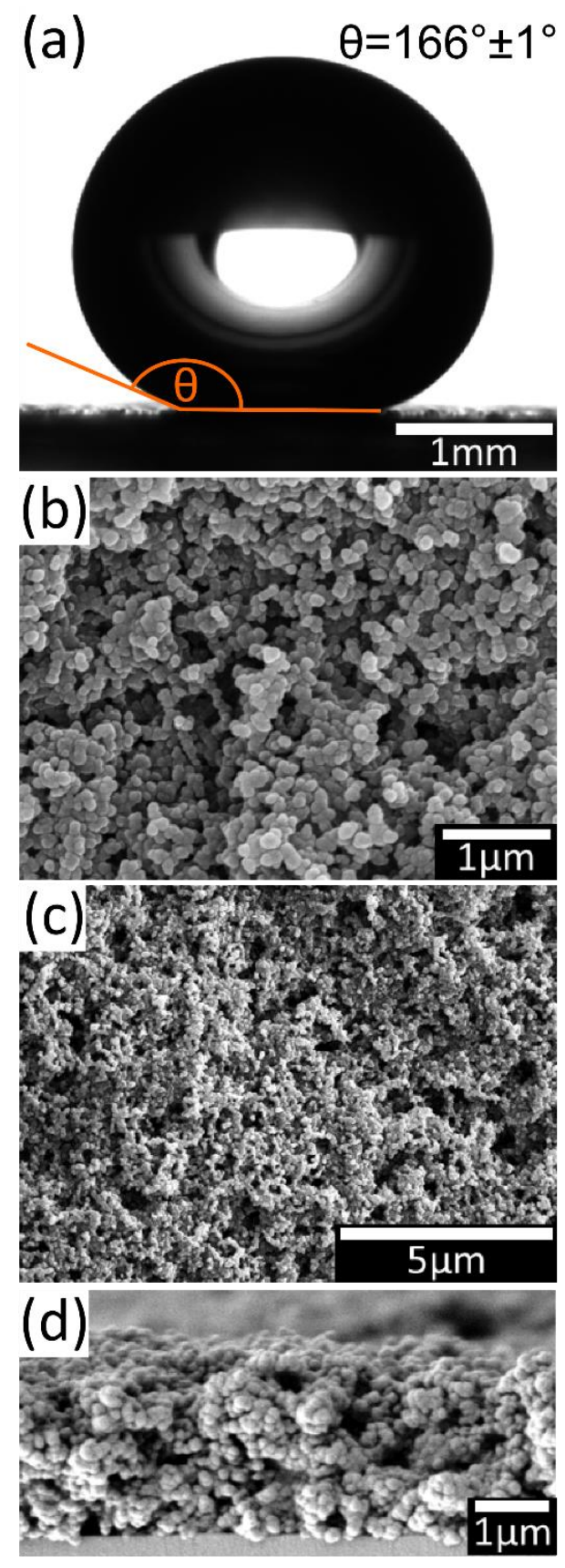

Fig 2. (a) A stationary $15 \mu$ d droplet of water on coated glass displaying a high static contact angle. (b) and (c) Scanning Electron microscope (SEM) image of the carbon nanoparticle surface displaying the aggregated clusters forming the porous microstructure of the coating. (D) Crosssection SEM image of the carbon nanoparticle coating. 


\section{Results and Disscussion}

Drop shape analysis was used to determine the wetting properties of the CNP spray coating. Fig 1(a) shows a $15 \mu 1$ droplet of distilled water on the surface of a glass slide that was coated with the CNP layer. Droplets on the surface display advancing, static, and receding contact angles of $167^{\circ} \pm 1^{\circ}, 166^{\circ} \pm 1^{\circ}$, and $164^{\circ} \pm 1^{\circ}$, respectively. The high contact angles and the low contact angle hysteresis of $3^{\circ}$, as indicated by the difference in the advancing and receding contact angles, indicate the presence of a Cassie-Baxter wetting state and demonstrates the superhydrophobicity of the surface. This allows for the low friction motion of water droplets on the surface of coated substrates. Fig 1(b) and 1(c) show top and cross section views of the CNP coating. The images show how the CNPs cluster and construct a network of microscopic cavities during the spray coating process, thus forming a multiscale rough surface. It is the combination of the hydrophobic carbon nanoparticles and the network of clustered particles that allows for the Cassie state and renders the surface superhydrophobic, as demonstrated by the measured contact angles. The small proportion of PDMS elastomer in the mixture acts as a binding agent, aiding the adhesion to the surface and improving the bond between the CNPs. This prevents the removal of soot particles when water flows over the surface as shown in figure 3(e), where water can be seen flowing over the surface of a \#400 stainless steel mesh that has been spray coated with the CNP mixture. The water is seen flowing over the mesh that is suspended in mid-air, without water penetrating the superhydrophobic porous surface and without removal of the CNPs from the surface.

Figure 3(a) and 3(b) show a \#400 stainless steel mesh that is uncoated (a) and coated (b). The coating forms an even layer on the solid metal wires of the mesh without filling the voids between the wires of the mesh. This was true for all the meshes tested and was confirmed with SEM analysis. The uncoated \#400 stainless steel mesh has an average wire diameter of $29 \pm 1 \mu \mathrm{m}$ and 
wire separation of $36 \pm 1 \mu \mathrm{m}$. After applying the CNP coating, the wire diameter increased to $31 \pm 1$ $\mu \mathrm{m}$, with a drop in the wire separation to $31 \pm 1 \mu \mathrm{m}$ due to the presence of the coating. The average thickness of the CNP coating for all the meshes was $2 \pm 1 \mu \mathrm{m}$. Again, the CNPs cluster and accumulate to form a porous structure with microscopic voids throughout the layer (Fig 3(d)). The network structure and the addition of the elastomer allows the coating to stretch and compress as the flexible mesh is shaped in order to form the U-shaped channels, and to still remain superhydrophobic under the deformation, as seen in figure 3(e).

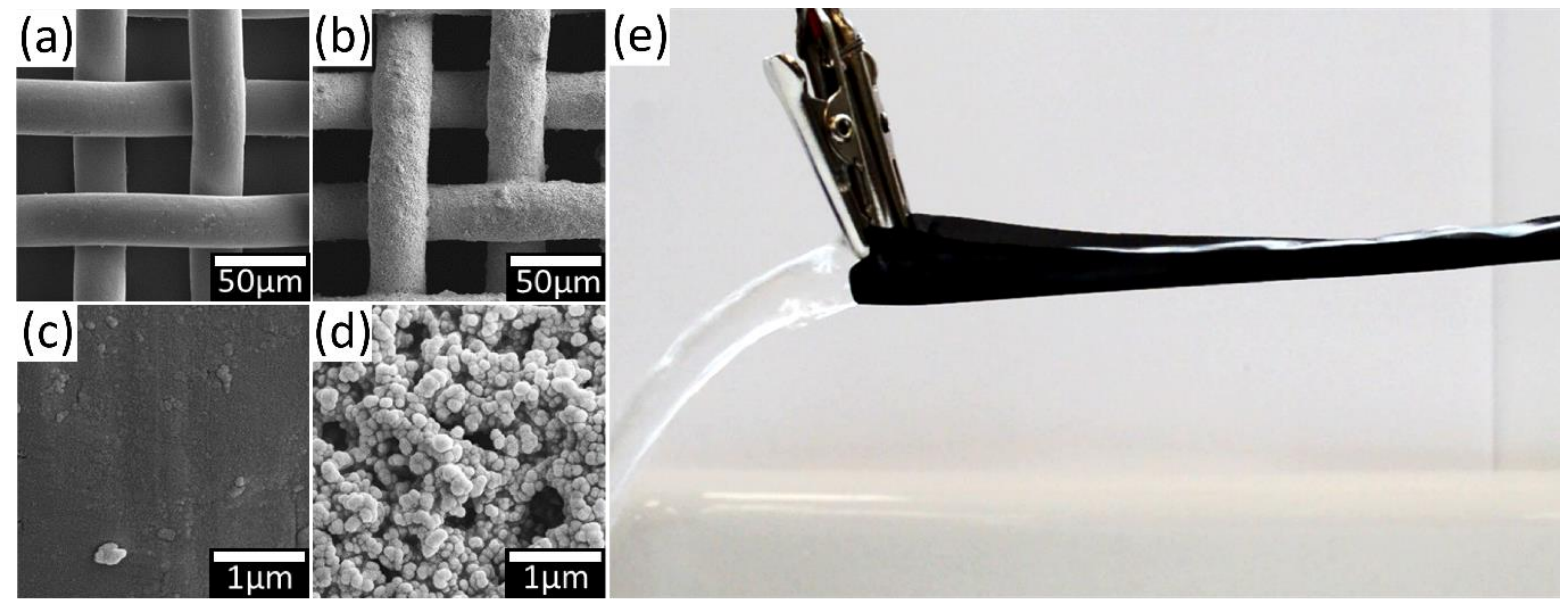

Fig 3. Images (a), (b), (c), and (d) show the difference between an uncoated and spray coated \#400 stainless steel mesh, respectively, at two magnifications. (e) Image of water being directed and flowing over a coated \#400 mesh U-shaped mesh channel.

To test the wetting properties of the meshes, drop shape analysis was performed. The results can be seen in Fig 4(b). All the uncoated meshes had consistently high advancing contact angles, between $129^{\circ}$ and $137^{\circ}$. Conversely, the receding contact angles for all the uncoated meshes were 
extremely low, and so considered to be $\sim 0^{\circ}$. This is a consequence of the water conforming to a Wenzel wetting state, with the water following over the contours of the wire in the mesh. Therefore, a droplet of water on the surface of the metallic mesh makes complete contact with the underlying solid area, and spreads over the surface. The adhesion of the liquid with the large solid surface area of the mesh results in the low receding angle.

After spray coating the \#150, \#250, \#400, and \#500 meshes became superhydrophobic, with both advancing and receding contact angles greater than $150^{\circ}$. The highest angles were recorded for the \#400 stainless steel mesh with advancing and receding contact angles of $164 \pm 2^{\circ}$ and $156 \pm 2^{\circ}$, respectively. The superhydrophobicity of the \#98 mesh was marginal, with advancing and receding contact angles of $157 \pm 2^{\circ}$ and $149 \pm 1^{\circ}$, respectively. The \#50 mesh remained hydrophobic after coating with advancing and receding contact angles of $153 \pm 3^{\circ}$ and $129 \pm 3^{\circ}$, respectively. This may be due to the liquid struggling to bridge between the wires of the larger meshes, \#50 and \#98, where the separation of the wires is $320 \pm 10 \mu \mathrm{m}$ and $192 \pm 4 \mu \mathrm{m}$, respectively.

The $20 \mathrm{cSt}$ silicone oil completely wicked into the uncoated and superhydrophobic mesh surfaces when drop shape analysis was performed. The contact angle was therefore considered $\sim 0^{\circ}$. This is considered superoleophilic.

The significant difference between the water and silicone oil contact angles on the CNP coated meshes demonstrates the difference in the wetting properties of the liquids. With the surface being superhydrophobic, superoleophilic, and porous, it makes a good choice for oil/water separation. 


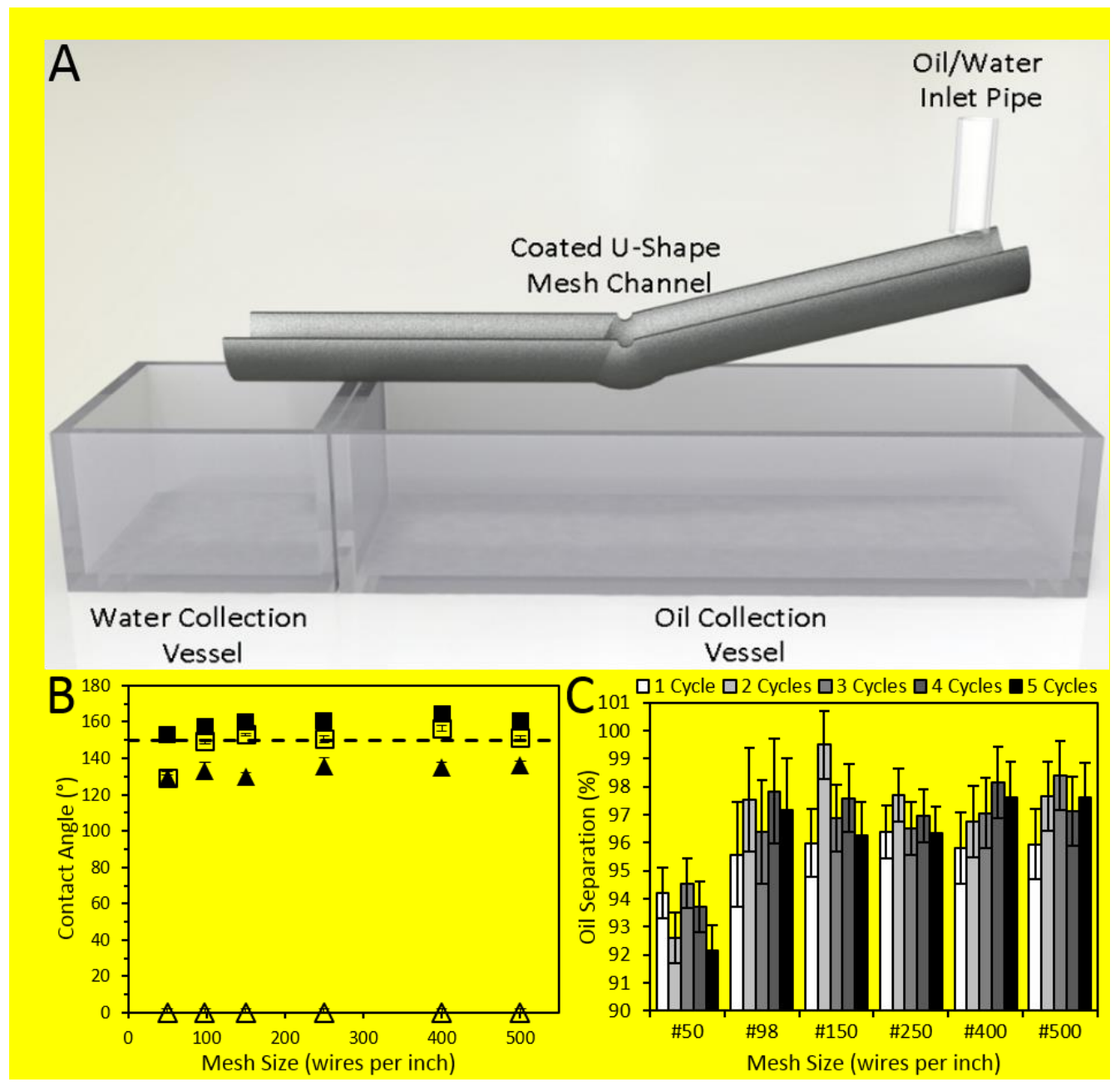

Fig 4. (a) Illustration of the oil/water separation setup (b) The advancing (filled) and receding (unfilled) contact angles for the six uncoated $(\boldsymbol{\Lambda}, \Delta)$ and coated $(\boldsymbol{\square}, \square)$ stainless steel meshes. The dashed line (---) indicates the minimum contact angle required, $150^{\circ}$, for a surface to be considered superhydrophobic. (c) The percentage of oil separated by each of the coated stainless steel meshes over 5 cycles of the experiment.

In Fig 4(a), an illustration of the oil/water separation experimental arrangement is presented. The $100 \mathrm{~mm}$ by $50 \mathrm{~mm}$ area of superhydrophobic mesh was folded and then unfolded along its major 
axis to form a central crease. The mesh was then rolled to form a hemi-cylindrical channel, with the crease pointing outward. The crease in the mesh acts as a low point in the channel, when mounted horizontally. Fig 3(e) shows a \#400 mesh that has been spray coated and formed into a u-shape channel. Water can be seen flowing along the channel and exiting the end of the channel, without penetrating the porous material. The ability to fold and conform to specific geometries shows the non rigid nature of the coating, that is not damaged when subjected to bending or folding.

As the oil water mixture was deposited onto the mesh, the silicone oil in the mixture wicked into the surface while the water remained on the surface. As the remainder of the mixture dripped onto the surface, the oil accumulated and flowed through and on the underside of the mesh. This eventually made its way to the crease, where it formed droplets that dripped into the oil collection vessel. Conversely, the droplets of water travelled over the top surface of the mesh until they reached the edge, where they fell into the water collection vessel. This continued until all the mixture had passed onto the coated mesh. The mesh was left for a further 2 minutes to allow any oil to drip into the oil collection vessel.

Fig 4(c) shows the results of the oil/water separation experiments. The percentage of oil collected from a single cycle over the mesh was calculated from the mass of the collected in the oil collection vessel and the mass of the oil in the original mixture. The same procedure was repeated in order to determine the separation percentage over 5 cycles with each of the coated mesh sizes. The \#50 coated mesh was the least capable of separating the oil from the mixture, with an average separation percentage of $93.4 \pm 1.0 \%$. All other coated meshes were capable of removing over $96 \%$ of the oil from the mixture. The \#500 coated mesh had the highest separation percentage, with an avergage separation of $97.4 \pm 0.9 \%$. 
The repeated cycle tests show that the oil separation percentage remains fairly constant over the 5 cycles, varying by $1.3 \%$ to $3.5 \%$. This implies that the surface is stable during the separation process and does not have any detrimental effects on the surface coating.

To improve the efficiency of the separation process, the length of the channel can be increased in order to increase the contact length over which the mixture can separate. This would allow more time for the constituent parts to separate. The calculated separation percentage values may also be lower than the true values as a portion of the oil is held in the porous structure of the superoleophilic mesh after the separation experiment has concluded. This implies that the true separation percentage is higher than the recorded value.

The flexibility of the surfaces and the ability to coat complex geometries and large areas means that this method can be scaled up in order to separate large volumes of oil/water mixtures.

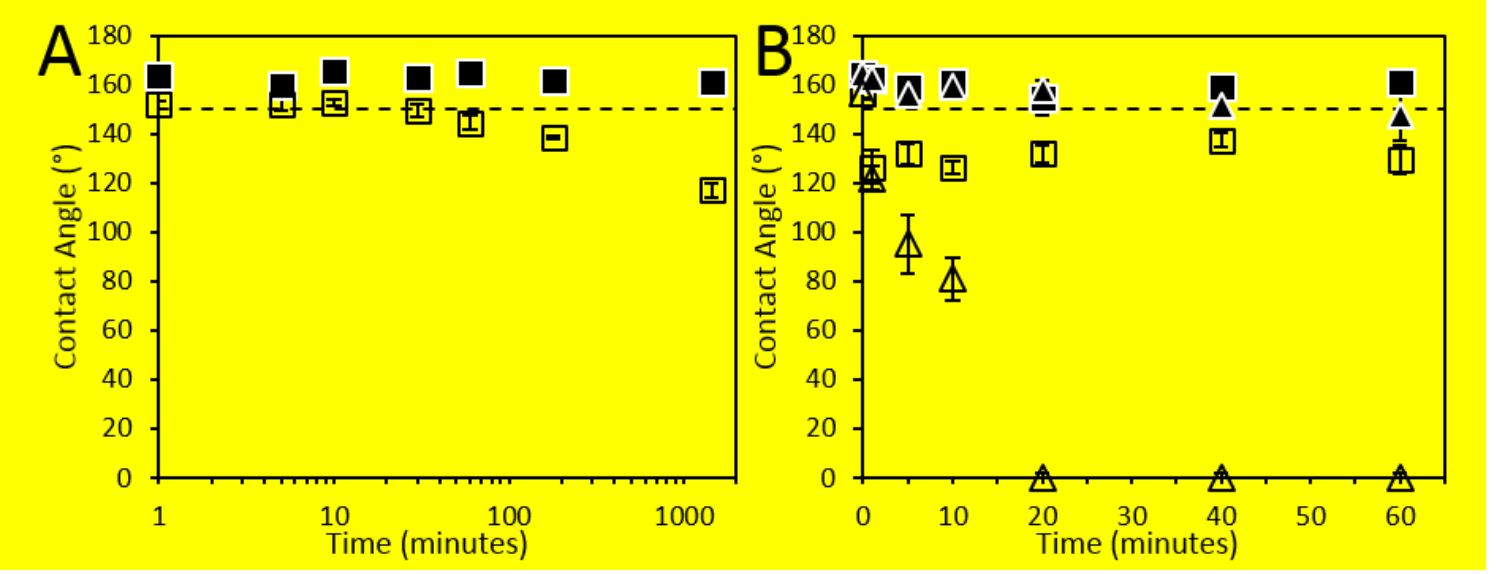

Fig 5.(a) shows the change over time of the advancing ( $\square)$ and receding( $\square)$ contact angles for a \#400 coated mesh exposed to $\mathrm{NaCl}$ solution (3.5\%wt). (b) shows the change over time of the advancing $(\bullet, \mathbf{\Lambda})$ and receding $(O, \Delta)$ contact angles for a $\# 400$ coated mesh exposed to $1 \mathrm{M} \mathrm{HCl}$ solution $(\boldsymbol{\Theta}, \bigcirc)$ and to $1 \mathrm{M} \mathrm{KOH}$ solution $(\boldsymbol{\Delta}, \Delta)$. The dashed line (--) marks the $150^{\circ}$ angle. 
Figure 5(a) shows the results of the $\mathrm{NaCl}$ stability test. The data shows that the advancing contact angle remains high, $\sim 160^{\circ}$, even after $24 \mathrm{hrs}$. The receding contact angle, however, remained above $150^{\circ}$ for $\sim 30 \mathrm{mins}$ and then gradually fell to $117.0 \pm 2.8^{\circ}$ after $24 \mathrm{hrs}$. The results of the acidic and basic environment tests are shown in fig. 5(b). In the $1 \mathrm{M} \mathrm{HCl}$ acidic environment, $\mathrm{pH}=0.34$, the advancing contact angle of the coated mesh remains above $150^{\circ}$, even after $1 \mathrm{hr}$, however, the receding contact angle instantly drops and plateaus around $130^{\circ}$. When exposed to the $1 \mathrm{M} \mathrm{KOH}$ basic environment, $\mathrm{pH}=13.46$, the advancing contact angle gradually falls to $147.3 \pm 9.8^{\circ}$ after 60 mins. There is a greater effect on the receding angle of the material, with the value falling to $0^{\circ}$ after 20 mins exposure to the basic environment.

In conclusion, the application of the CNP spray coating onto a surface renders the surface superhydrophobic, displaying high static contact angles of $164 \pm 1^{\circ}$ and low hysteresis, when applied to a flat surface. The dual scale roughness of the surface, with micro and nano scale features are present, acts in similar a way to that of the Sacred Lotus, in order to render the surface superhydrophobic. The spray coating, consisting of carbon nanoparticles derived from soot, toluene, and PDMS is inexpensive and simple to produce, due to the low cost of the materials and the simple application method. The application method also allows for large area coverage, in this instance of $0.5 \mathrm{~m}^{2}$, and the coating of complex geometries such as meshes, which display contact angles of $\sim 160^{\circ}$ for a coated \#400 stainless steel mesh. We have observed on some flat surfaces tests that it is possible remove the baking step in the process, which results in a much longer curing time for the elastomer in the mixture. This removes the constraints on size of object that may be covered. The ability to coat flexible conformable surfaces allows for the formation of channels or tubes that can be used to direct liquid flows. The use of coated meshes offers a way to separate oil/water mixtures due to the very different wetting properties of the two liquids on the porous 
surface. When tested, the majority of the meshes were capable of separating out over $96 \%$ of the oil from the mixture during cycle tests, as the mixture flowed along the coated mesh channels.

\section{Acknowledgments}

The work was supported by the UK Engineering and Physical Sciences Research Council (EPSRC) through grants EP/ L026899/1, EP/L026619/1, and EP/L026341/1. The authors would also like to thank Professor James Martin and Dr. Simone Stuart-Cole from Reece Innovation, Newcastle-upon-Tyne for their input and guidance.

\section{References}

[1] Solga A, Cerman Z, Striffler B F, Spaeth M and Barthlott W 2007 The dream of staying clean: Lotus and biomimetic surfaces Bioinspiration \& Biomimetics 2 S126-34

[2] Koch K and Barthlott W 2009 Superhydrophobic and superhydrophilic plant surfaces: an inspiration for biomimetic materials Philosophical Transactions of the Royal Society A: Mathematical, Physical and Engineering Sciences 367 1487-509

[3] Barthlott W and Neinhuis C 1997 Purity of the sacred lotus, or escape from contamination in biological surfaces Planta 202 1-8

[4] Young T 1805 An Essay on the Cohesion of Fluids Philosophical Transactions of the Royal Society of London 95 65-87 
[5] Wenzel R N 1936 Resistance Of Solid Surfaces To Wetting By Water Industrial \& Engineering Chemistry 28 988-94

[6] Cassie A B D and Baxter S 1944 Wettability of porous surfaces Transactions of the Faraday Society $\mathbf{4 0} 546$

[7] Shirtcliffe N J, McHale G, Atherton S and Newton M I 2010 An introduction to superhydrophobicity Advances in Colloid and Interface Science 161 124-38

[8] Onda T, Shibuichi S, Satoh N and Tsujii K 1996 Super-Water-Repellent Fractal Surfaces Langmuir 12 2125-7

[9] Ma M, Hill R M, Lowery J L, Fridrikh S V and Rutledge G C 2005 Electrospun Poly(Styrene- block -dimethylsiloxane) Block Copolymer Fibers Exhibiting Superhydrophobicity Langmuir 21 5549-54

[10] Shirtcliffe N J, McHale G, Newton M I and Perry C C 2003 Intrinsically Superhydrophobic Organosilica Sol-Gel Foams Langmuir 19 5626-31

[11] Shibuichi S, Onda T, Satoh N and Tsujii K 1996 Super Water-Repellent Surfaces Resulting from Fractal Structure The Journal of Physical Chemistry 100 19512-7

[12] Shirtcliffe N J, McHale G, Newton M I and Perry C C 2005 Wetting and Wetting Transitions on Copper-Based Super-Hydrophobic Surfaces Langmuir 21 937-43

[13] Shirtcliffe N J, McHale G, Newton M I, Chabrol G and Perry C C 2004 Dual-Scale Roughness Produces Unusually Water-Repellent Surfaces Advanced Materials 161929 
[14] Shirtcliffe N J, Aqil S, Evans C, McHale G, Newton M I, Perry C C and Roach P 2004 The use of high aspect ratio photoresist (SU-8) for super-hydrophobic pattern prototyping Journal of Micromechanics and Microengineering 14 1384-9

[15] Lee W, Jin M-K, Yoo W-C and Lee J-K 2004 Nanostructuring of a polymeric substrate with well-defined nanometer-scale topography and tailored surface wettability. Langmuir $207665-9$

[16] Brennan J C, Geraldi N R, Morris R H, Fairhurst D J, McHale G and Newton M I 2015 Flexible conformable hydrophobized surfaces for turbulent flow drag reduction Scientific Reports 510267

[17] Ogihara H, Xie J, Okagaki J and Saji T 2012 Simple Method for Preparing Superhydrophobic Paper: Spray-Deposited Hydrophobic Silica Nanoparticle Coatings Exhibit High Water-Repellency and Transparency Langmuir 28 4605-8

[18] Ogihara H, Okagaki J and Saji T 2011 Facile Fabrication of Colored Superhydrophobic Coatings by Spraying a Pigment Nanoparticle Suspension Langmuir 27 9069-72

[19] Latthe S S and Rao A V 2012 Superhydrophobic SiO2 micro-particle coatings by spray method Surface and Coatings Technology 207 489-92

[20] Zhang Y, Ge D and Yang S 2014 Spray-coating of superhydrophobic aluminum alloys with enhanced mechanical robustness Journal of Colloid and Interface Science 423 101-7

[21] Li J, Yan L, Li H, Li J, Zha F and Lei Z 2015 A facile one-step spray-coating process for the fabrication of a superhydrophobic attapulgite coated mesh for use in oil/water 
separation RSC Adv. $553802-8$

[22] Zeiger C, Rodrigues da Silva I C, Mail M, Kavalenka M N, Barthlott W and Hölscher H 2016 Microstructures of superhydrophobic plant leaves - inspiration for efficient oil spill cleanup materials Bioinspiration \& Biomimetics 1156003

[23] O’Loughlin T E, Martens S, Ren S R, McKay P and Banerjee S 2017 Orthogonal Wettability of Hierarchically Textured Metal Meshes as a Means of Separating Water/Oil Emulsions Advanced Engineering Materials 1600808

[24] Zhou X, Zhang Z, Xu X, Guo F, Zhu X, Men X and Ge B 2013 Robust and durable superhydrophobic cotton fabrics for oil/water separation ACS Applied Materials and Interfaces 5 7208-14

[25] Kwon G, Kota A K, Li Y, Sohani A, Mabry J M and Tuteja A 2012 On-demand separation of oil-water mixtures Advanced Materials 24 3666-71

[26] Crick C R, Gibbins J A and Parkin I P 2013 Superhydrophobic polymer-coated coppermesh; membranes for highly efficient oil-water separation Journal of Materials Chemistry A 15943

[27] Crick C R, Ozkan F T and Parkin I P 2015 Fabrication of optimized oil-water separation devices through the targeted treatment of silica meshes Science and Technology of Advanced Materials 1655006

[28] Liu M, Hou Y, Li J and Guo Z 2017 Stable Superwetting Meshes for On-Demand Separation of Immiscible Oil/Water Mixtures and Emulsions Langmuir 
acs.langmuir. $7 \mathrm{~b} 00658$

[29] Zhang F, Zhang W Bin, Shi Z, Wang D, Jin J and Jiang L 2013 Nanowire-haired inorganic membranes with superhydrophilicity and underwater ultralow adhesive superoleophobicity for high-efficiency oil/water separation Advanced Materials 254192 8

[30] Song J, Huang S, Lu Y, Bu X, Mates J E, Ghosh A, Ganguly R, Carmalt C J, Parkin I P, Xu W and Megaridis C M 2014 Self-driven one-step oil removal from oil spill on water via selective-wettability steel mesh ACS Applied Materials and Interfaces 6 19858-65

[31] Deng D, Prendergast D P, MacFarlane J, Bagatin R, Stellacci F and Gschwend P M 2013 Hydrophobic meshes for oil spill recovery devices ACS Applied Materials and Interfaces $5774-81$

[32] Liu Y, Zhang K, Yao W, Liu J, Han Z and Ren L 2016 Bioinspired structured superhydrophobic and superoleophilic stainless steel mesh for efficient oil-water separation Colloids and Surfaces A: Physicochemical and Engineering Aspects 500 54-63

[33] Gupta P and Kandasubramanian B 2017 Directional Fluid Gating by Janus Membranes with Heterogeneous Wetting Properties for Selective Oil/Water Separation ACS Applied Materials \& Interfaces 9 19102-13

[34] Zhang X, Li Z, Liu K and Jiang L 2013 Bioinspired Multifunctional Foam with SelfCleaning and Oil/Water Separation Advanced Functional Materials 23 2881-6

[35] Li J, Yan L, Tang X, Feng H, Hu D and Zha F 2016 Robust Superhydrophobic Fabric 
Bag Filled with Polyurethane Sponges Used for Vacuum-Assisted Continuous and Ultrafast Absorption and Collection of Oils from Water Advanced Materials Interfaces 3 1500770

[36] Li J, Xu C, Zhang Y, Wang R, Zha F and She H 2016 Robust superhydrophobic attapulgite coated polyurethane sponge for efficient immiscible oil/water mixture and emulsion separation J. Mater. Chem. A 4 15546-53

[37] Li J, Li D, Yang Y, Li J, Zha F and Lei Z 2016 A prewetting induced underwater superoleophobic or underoil (super) hydrophobic waste potato residue-coated mesh for selective efficient oil/water separation Green Chem. 18 541-9

[38] Deng X, Mammen L, Butt H-J and Vollmer D 2012 Candle soot as a template for a transparent robust superamphiphobic coating. Science (New York, N.Y.) 335 67-70

[39] Brennan J C, Geraldi N R, Morris R H, Fairhurst D J, McHale G and Newton M I 2015 Flexible conformable hydrophobized surfaces for turbulent flow drag reduction Scientific Reports 510267

[40] Geraldi N R, Ouali F F, Morris R H, McHale G and Newton M I 2013 Capillary origami and superhydrophobic membrane surfaces Applied Physics Letters 102214104

[41] Geraldi N R 2015 Wetting of Non-Rigid Surfaces (Nottingham Trent University) 